\title{
Optimización en sistemas de control multifrecuencia
}

\author{
E. Aranda-Escolástico ${ }^{1}$, J. Salt ${ }^{2}$, M. Guinaldo ${ }^{1}$, J. Chacón ${ }^{1}$, H. $\operatorname{Vargas}^{3}$, S. Dormido ${ }^{1}$ \\ ${ }^{1}$ Dpt. Informática y Automática, Universidad Nacional de Educación a Distancia, Madrid, España \\ earandae@bec.uned.es, mguinaldo@dia.uned.es, jchacon@bec.uned.es, sdormido@dia.uned.es \\ ${ }^{2}$ Dpt. de Ingeniería de Sistemas y Automática, Universitat Politècnica de València, Valencia, España \\ julian@isa.upv.es \\ ${ }^{3}$ Pontificia Universidad Católica de Valparaíso, Valparaíso, Chile \\ hector.vargas@pucv.cl
}

\section{Resumen}

En este trabajo consideramos una configuración multifrecuencia en la que $n$ señales de control se calculan y aplican al sistema en cada periodo de muestreo de la salida de la planta. Nuestro objetivo es maximizar el radio de convergencia del sistema a la referencia deseada. Para ello, diseñamos un algoritmo de optimización en función de las $n$ señales de control y/o de los tiempos de aplicación de dichas señales. Para verificar la validez del algoritmo, presentamos algunos resultados experimentales obtenidos en un sistema de levitación por aire.

Palabras clave: Sistemas multifrecuencia, optimización, sistema de levitación por aire

\section{INTRODUCCIÓN}

Este artículo se enmarca en el contexto de los sistemas multifreciencia. Concretamente, consideramos un sistema de dos frecuencias donde la salida del sistema se muestra con un periodo de muestreo $T_{s}$ mientras que la entrada calculada por el controlador se aplica más rápidamente. Tradicionalmente, si el controlador transmite $n$ señales de control, estas señales se aplican, cada una, durante $T_{s} / n$ unidades de tiempo (véase, por ejemplo, [25, 29]). En algunos casos, los tiempos de aplicación de las señales de control pueden ser irregulares $[1,8,9]$ pero no existen en la literatura criterios concretos para decidir esta irregularidad.

Los sistemas de control multifrecuencia se han estudiado intensamente en la literatura $[14,16,4]$, ya que presentan múltiples aplicaciones. Por ejemplo, el control multifrecuencia puede utilizarse en analizadores químicos $[20,27]$, realimentación visual $[23,30]$, control de vuelo [13], o en la lectura y escritura de discos duros [33, 28]. También se ha mostrado útil su aplicación a sistemas de control en red $[26,21,9]$, proporcionando un ahorro de recursos de comunicación al obtener una respuesta similar al caso monofrecuencia con una cantidad reducida de información. Desde el punto de vista del problema de optimización, se han desarrollado diferentes controladores óptimos en la literatura. En [17] se describe un control óptimo $H_{2}$. En [31] se propone un control óptimo periódico. Por su parte, en [18], se diseña un controlador óptimo basado en optimización de desigualdades matriciales lineales. Además, se han propuesto múltiples controladores predictivos en este marco $[19,11,5]$. Sin embargo, incluso cuando estos trabajos proponen diferentes controladores óptimos, no se considera en ninguno de ellos la posibilidad de muestreos irregulares o de retardos variables en el tiempo. Recientemente, estrategias como el control de mínima atención $[2,10]$ y el control con atención en cualquier momento $[12,24]$ se han estudiado en el marco de los sistemas de control en red. En el control de mínima atención, el tiempo de aplicación de la señal de control se maximiza mientras se mantiene un cierto nivel de desempeño. En el control con atención en cualquier momento, el tiempo de aplicación se fija de manera previa en función de los recursos disponibles y se maximiza el desempeño durante dicho periodo. El método que proponemos en este trabajo trata, de alguna manera, de combinar ambas ideas. Por un lado, se maximiza el radio de convergencia del sistema tras un periodo completo de la salida, que es fijo. Sin embargo, dentro de este intervalo de tiempo, se permite la variación de los tiempos de aplicación de la señal de control no solo para mantener el comportamiento sino para mejorarlo.

El resto del trabajo está organizado de la siguiente manera. En la sección 2 se presenta el problema y el modelo multifrecuencia. En la sección 3 se describe el algoritmo de optimización, así como algunas técnicas para la implementación en sistemas con restricciones computacionales. La sección 4 recoge los resultados experimentales obtenidos en el sistema de levitación por aire. Finalmente, en la sección 5 se recoge una discusión sobre los beneficios del método propuesto frente a otros sistemas de la literatura.

\section{Preliminares}

Definimos el conjunto de los números reales y el conjunto de números naturales como $\mathbb{R}$ y $\mathbb{N}$, respectivamente. El espacio real $n$-dimensional se de- 
fine por $\mathbb{R}^{n}$. Nos referimos a la norma euclídea del vector $x \in \mathbb{R}^{n}$ como $\|x\|=\sqrt{x^{\top} x}$. Sea $M \in$ $\mathbb{R}^{n \times m} ; M^{\top}$ denota la matriz transpuesta de $M$. Además, si $M$ es una matriz real simétrica, entonces el máximo y el mínimo autovalor de $M$ se denotan por $\lambda_{\text {máx }}(M)$ y $\lambda_{\text {mín }}(M)$, respectivamente. Por otra parte, denotamos una matriz simétrica positiva-definida $P \in \mathbb{R}^{n \times n}$ como $P>0$, mientras $P \geq 0, P<0$, y $P \leq 0$ hacen referecia a matrices simétrica positiva-semidefinida, negativa-definida, y negativa-semidefinida, respectivamente. Denotamos la matriz identidad $\mathbb{I} \in \mathbb{R}^{n \times n}$ por $\mathbb{I}_{n}$. Sea $A \in \mathbb{R}^{n \times n}$ y $B \in \mathbb{R}^{n \times m}$, definimos $\mu(A)=$ máx $\left\{\mu \mid \mu \in \lambda\left(\left(A+A^{\top}\right) / 2\right)\right\}$. Además, la norma de la matriz exponencial [22] puede acotarse de la siguiente forma

$$
\left\|e^{A \theta}\right\| \leq e^{\mu(A) \theta} \leq e^{\mu(A)(\theta+\epsilon)}, \forall \epsilon \geq 0 .
$$

$B(\theta, A)$ denota

$$
B(\theta, A)=\left\{\begin{array}{cc}
0 & \text { si } \theta \leq 0 \\
\int_{0}^{\theta} e^{A s} B d s & \text { si } \theta>0
\end{array}\right.
$$

y $B_{\mu}(\theta, A)$

$$
B_{\mu}(\theta, A)=\left\{\begin{array}{cl}
0 & \text { si } \theta \leq 0 \\
\int_{0}^{\theta} e^{\mu(A) s}\|B\| d s & \text { si } \theta>0
\end{array} .\right.
$$

Consecuentemente,

$$
\|B(\theta, A)\| \leq B_{\mu}(\theta, A) \leq B_{\mu}(\theta+\epsilon, A)
$$

para todo $\epsilon \geq 0$. Finalmente, definimos la estabilidad exponencial de un sistema

$$
\dot{x}(t)=f(t, x)
$$

donde $f:[0, \infty) \times D \rightarrow \mathbb{R}^{n}$ es continua a trozos en $t$ y localmente lipschitziana en $x$ en $[0, \infty) \times D$, y donde $D \in \mathbb{R}^{n}$ es un dominio que contiene el origen $x=0$, como sigue [15]:

Definición 1. El punto de equilibrio $x=0$ de (4) es exponencialmente estable si existen constantes positivas $\epsilon, c, \mathrm{y} \alpha$ tales que

$$
\|x(t)\| \leq c e^{-\alpha\left(t-t_{0}\right)}\left\|x\left(t_{0}\right)\right\|, \forall\left\|x\left(t_{0}\right)\right\|<\epsilon
$$

y globalmente exponencialmente estable si (5) se satisface para cualquier estado inicial $x\left(t_{0}\right)$.

\section{PLANTEAMIENTO DEL PROBLEMA}

Considérese el sistema continuo lineal e invariante en el tiempo (linear time-invariant, LTI)

$$
\begin{aligned}
& \dot{x}_{p}(t)=A_{p} x_{p}(t)+B_{p} u(t) \\
& y(t)=C_{p} x_{p}(t)
\end{aligned}
$$

siendo $x_{p}(t) \in \mathbb{R}^{n_{x_{p}}}$ el vector de estados de la planta, $u(t) \in \mathbb{R}^{n_{u}}$ el vector de entrada, $y(t) \in \mathbb{R}^{n_{y}}$ el vector de salida, y $A_{p} \in \mathbb{R}^{n_{x_{p}} \times n_{x_{p}}}, B_{p} \in \mathbb{R}^{n_{x_{p}} \times n_{u}}$, y $C_{p} \in \mathbb{R}^{n_{y} \times n_{x_{p}}}$ matrices constantes. Asumimos que la salida de (6) se muestrea con periodo $T_{s}$, tal y como se muestra en la figura 1. Por otra parte, el controlador cambia la señal de control $n$ veces durante $T_{s}$. Asumimos también que el valor de la entrada es $u_{i}$ con $i=1, \ldots, n$ durante el periodo de tiempo $T_{i}$, cumpliéndose $T_{s}=\sum_{i=1}^{n} T_{i}$. Por tanto,

$$
u(t)=\left\{\begin{array}{c}
u_{1} \text { if } t \in\left[k T_{s}, k T_{s}+T_{1}\right) \\
u_{2} \text { if } t \in\left[k T_{s}+T_{1}, k T_{s}+T_{1}+T_{2}\right) \\
\vdots \\
u_{n} \text { if } t \in\left[k T_{s}+\sum_{i=1}^{n-1} T_{i}, k T_{s}+\sum_{i=1}^{n} T_{i}\right)
\end{array} .\right.
$$

La discretización de (6)-(7) da lugar a un sistema discreto, periódico, lineal y variable en el tiempo, lo que implica diferentes problemas a la hora de garantizar la estabilidad y, en consecuencia, maximizar el radio de convergencia. Una manera de evitar esto es haciendo uso de las técnicas de estiramiento (lifting) $[16,4]$. Así pues, podemos escribir (6)-(7) de forma estirada como

$$
x_{p}(t)=e^{A_{p}\left(t-T_{s}\right)} x_{p}(k)+\sum_{i=1}^{n} B_{p}^{i}\left(t-T_{s}, A_{p}\right) u_{i}
$$

con

$$
\begin{aligned}
B_{p}^{i}\left(t-T_{s}, A_{p}\right) & =e^{A_{p}\left(t-T_{s}-\operatorname{mín}\left(t-T_{s}, \sum_{j=0}^{i} T_{j}\right)\right)} \\
& \times B_{p}\left(\operatorname{mín}\left(t-T_{s}-\sum_{j=0}^{i-1} T_{j}, T_{i}\right), A_{p}\right) .
\end{aligned}
$$

Consecuentemente, el estado después de un periodo de muestreo $T_{s}$ es

$$
x_{p}\left((k+1) T_{s}\right)=\tilde{A}_{p} x_{p}\left(k T_{s}\right)+\tilde{B}_{p} U
$$

con $U=\left[\begin{array}{lll}u_{1} & \cdots & u_{n}\end{array}\right]^{\top}, \tilde{A}_{p}=e^{A_{p} T_{s}}$, y $\tilde{B}_{p}=$ $\left[B_{p}^{1}\left(T_{s}, A_{p}\right) \quad \cdots \quad B_{p}^{n}\left(T_{s}, A_{p}\right)\right]$.

\section{ALGORITMO DE OPTIMIZACIÓN}

En esta sección, proponemos el diseño de un algoritmo para maximizar el radio de convergencia del sistema. A priori, el radio de convergencia puede maximizarse tanto como se desee si la señal de entrada es lo suficientemente grande. Naturalmente, esto no es una solución apropiada para el sistema real, donde el actuador puede saturar la 


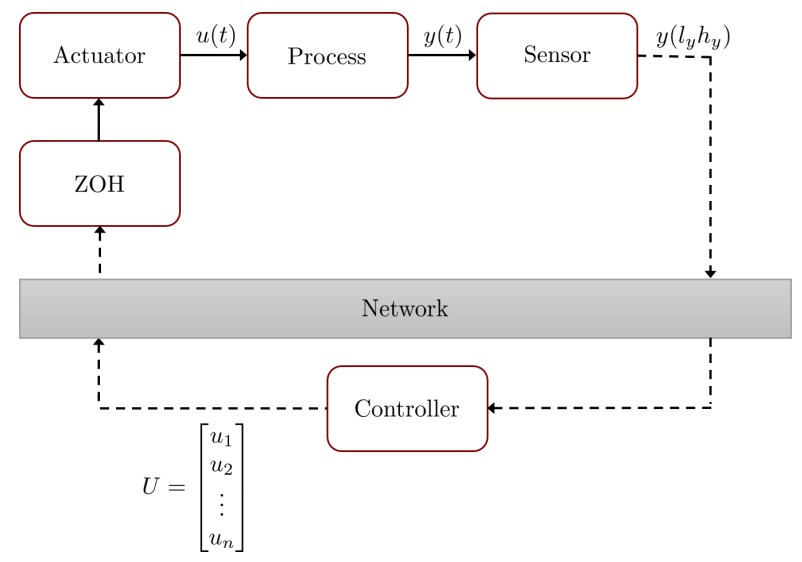

Figura 1: Diagrama de bloques de un sistema bifrecuencia con entrada rápida.

señal de entrada o no permitir cambios lo suficientemente rápidos. Para abordar este problema, consideramos un controlador auxiliar LTI para el que garantizamos la estabilidad asintótica global con una ganancia máxima, A continuación, proporcionamos las restricciones necesarias al problema de optimización para maximizar el radio de convergencia a la par que se mantiene esta ganancia máxima. Las pruebas de los teoremas se omiten por brevedad.

Para asegurar la estabilidad exponencial del sistema, hacemos uso del siguiente lema.

Lema 1. La estabilidad exponencial del sistema discretizado (10) está garantizada con radio de convergencia $\hat{\alpha}>0$, si existe una función de Lyapunov $V\left(x_{p}\left(k T_{s}\right)\right)=V(k): \mathbb{R}^{n_{x_{p}}} \rightarrow \mathbb{R}$, escalares positivos $\lambda_{1}, \lambda_{2}, y$ un entero positivo $q$ tales que

$$
\lambda_{1}\left\|x_{p}(k)\right\|^{q} \leq V(k) \leq \lambda_{2}\left\|x_{p}(k)\right\|^{q}
$$

$y$

$$
V(k+1)-e^{q \hat{\alpha} T_{s}} V(k) \leq 0 .
$$

Paralelamente, para calcular las señales de entrada $\left\{u_{i}\right\}$ y sus respectivos tiempos de aplicación $\left\{T_{i}\right\}$, necesitamos una estimación del modelo de la planta con el que obtener un conjunto de salidas estimadas $\left\{y_{p m, i}\right\}$. Considérese que el modelo de la planta está descrito por el estado estimado $x_{p m}$, y que se definen las matrices $A_{p m}, B_{p m}, C_{p m}$ análogamente a (6). Entonces, la salida estimada es

$$
\begin{aligned}
& y_{p m, 1}=y_{p}\left(k T_{s}\right) \text { para } t \in\left[k T_{s},(k+1) T_{s}\right) \\
& y_{p m, i}=C_{p m} x_{p m}\left(k T_{s}+\sum_{j=0}^{i-1} T_{j}\right) \\
& \quad \text { para } t \in\left[k T_{s},(k+1) T_{s}\right) \text { and } 1<i \leq n .
\end{aligned}
$$

Consideremos ahora que existe un controlador LTI auxiliar tal que

$$
\begin{aligned}
\dot{x}_{c}(t) & =A_{c} x_{c}(t)+B_{c} e(t) \\
\hat{u}(t) & =C_{c} x_{c}(t)+D_{c} e(t),
\end{aligned}
$$

donde $e(t)=r(t)-y(t)$, con $r(t)$ siendo la señal de referencia (tomamos $r(t)=0$ por simplicidad), $x_{c}(t) \in \mathbb{R}^{n_{x_{c}}}$ el vector de estados del controlador, $\hat{u}(t) \in \mathbb{R}^{n_{u}}$ el vector de entradas auxiliares, y $A_{c} \in \mathbb{R}^{n_{x_{c}} \times n_{x_{c}}}, B_{c} \in \mathbb{R}^{n_{x_{c}} \times n_{y}}, C_{c} \in \mathbb{R}^{n_{u} \times n_{x_{c}}}$ y $D_{c} \in \mathbb{R}^{n_{u} \times n_{y}}$ matrices constantes. En un escenario multifrecuencia y utilizando las técnicas de estiramiento, se puede escribir el lazo de control cerrado del estado aumentado $\hat{x}^{\top}=\left[\begin{array}{ll}x_{p} & x_{c}\end{array}\right]^{\top}$ como

$$
\hat{x}\left((k+1) T_{s}\right)=\Pi\left(\hat{T}_{1}, \ldots, \hat{T}_{n}\right) \hat{x}\left(k T_{s}\right),
$$

donde $\hat{T}_{i}$ es el tiempo de aplicación de $\hat{u}_{i}, \mathrm{y}$

$$
\begin{aligned}
& \Pi\left(\hat{T}_{1}, \ldots, \hat{T}_{n}\right)=e^{A T_{s}}+e^{A\left(T_{s}-\hat{T}_{1}\right)} B\left(\hat{T}_{1}, A\right) K \\
& \quad+\sum_{i=2}^{n} e^{A\left(T_{s}-\sum_{j=1}^{i} \hat{T}_{j}\right)} B\left(\hat{T}_{i}, A\right) K_{m} \\
& \quad \times \prod_{j=1}^{i}\left(e^{A_{m} \hat{T}_{i-j}}+B_{m}\left(\hat{T}_{i-j}, A_{m}\right) K_{m}\right)
\end{aligned}
$$

$A=\left[\begin{array}{cc}A_{p} & 0 \\ 0 & A_{c}\end{array}\right], \quad B=\left[\begin{array}{cc}B_{p} & 0 \\ 0 & B_{c}\end{array}\right], \quad K=$ $\left[\begin{array}{cc}-D_{c} C_{p} & C_{c} \\ -C_{p} & 0\end{array}\right], \quad A_{m}=\left[\begin{array}{cc}A_{p m} & 0 \\ 0 & A_{c}\end{array}\right], \quad B_{m}=$ $\left[\begin{array}{cc}B_{p m} & 0 \\ 0 & B_{c}\end{array}\right], K_{m}=\left[\begin{array}{cc}-D_{c} C_{p m} & C_{c} \\ -C_{p m} & 0\end{array}\right]$ y $\hat{T}_{0}=T_{0}=$

Para garantizar la estabilidad de (14), introducimos la siguiente hipótesis.

Hipótesis 1. Existen matrices $C_{c}$ y $D_{c}$, una matriz definida positiva $P$, y escalares $\hat{T}_{1}, \ldots, \hat{T}_{n} \geq 0$ tales que

$$
\begin{gathered}
\sum_{i=1}^{n} \hat{T}_{n}=T_{s} \\
\Pi^{\top}\left(\hat{T}_{1}, \ldots, \hat{T}_{n}\right) P \Pi\left(\hat{T}_{1}, \ldots, \hat{T}_{n}\right)-e^{-2 \hat{\alpha} T_{s}} P \leq 0,
\end{gathered}
$$

se satisfacen para algún $\hat{\alpha}>0$.

Finalmente, se puede formular el siguiente teorema

Teorema 3.1. Considérese el sistema auxiliar discretizado en lazo cerrado (14). Asúmase que la hipótesis 1 se cumple. Entonces, (14) es global y exponencialmente estable con, al menos, un radio 
de convergencia $\hat{\alpha}>0$ y una ganancia máxima

$$
\begin{aligned}
& c\left(\hat{T}_{1}, \ldots, \hat{T}_{n}\right)=\sqrt{\frac{\lambda_{\text {máx }}(P)}{\lambda_{\text {mín }}(P)}} e^{\hat{\alpha} T_{s}}\left(e^{\mu(A) T_{s}}\right. \\
& +e^{\mu(A)\left(T_{s}-\hat{T}_{1}\right)} B_{\mu}\left(\hat{T}_{1}, A\right)\|K\| \\
& +\sum_{i=2}^{n} e^{\mu(A)\left(T_{s}-\sum_{j=1}^{i} \hat{T}_{j}\right)} B_{\mu}\left(\hat{T}_{i}, A_{p}\right)\left\|K_{m}\right\| \\
& \left.\quad \times \prod_{j=1}^{i}\left(e^{\mu\left(A_{m}\right) \hat{T}_{i-j}}+B_{m \mu}\left(\hat{T}_{i-j}, A_{m}\right)\left\|K_{m}\right\|\right)\right) .
\end{aligned}
$$

Probado que existe un controlador capaz de estabilizar global y exponencialmente (14), podemos diseñar las señales de entrada y sus tiempos de aplicación para maximizar el radio de convergencia de system (10). Como se ha mencionado anteriormente, maximizar el radio de convergencia puede implicar aumentar la ganancia. Así pues, necesitamos establecer restricciones para mantener $c$ en valores aceptables. Considérese, por tanto, las siguientes hipótesis, que tienen por objetivo garantizar la optimización del radio de convergencia mientras se satisfacen las restricciones.

Hipótesis 2. Es posible calcular $\bar{c}$ tal que $\bar{c}=$ $\operatorname{máx}\left(c\left(\hat{T}_{1}, \ldots, \hat{T}_{n}\right)\right)$.

Hipótesis 3. El conjunto de señales de control $\left\{u_{i}\right\}$ para $i=1, \ldots, n$ satisface que

$$
\begin{aligned}
\left\|u_{1}\right\| & \leq\|K\|\left\|x\left(k T_{s}\right)\right\| \\
\left\|u_{i}\right\| & \leq\left\|K_{m}\right\|\left\|x_{m}\left(k T_{s}+\sum_{j=0}^{i-1} T_{j}\right)\right\|, \text { for } i>1
\end{aligned}
$$

Entonces, el siguiente teorema puede formularse.

Teorema 3.2. Considérese que el sistema linear discretizado en lazo cerrado (10) con señales de control $\left\{u_{i}\right\}$ aplicadas durante periodos $\left\{T_{i}\right\}$ for $i=1, \ldots, n$. Asúmase que las hipótesis 2 y 3 se mantienen. Entonces, el sistema (10) es global y exponencialmente estable con, al menos, radio de convergencia $\alpha \geq \hat{\alpha} y$ ganancia $\bar{c}$.

Nota 1. Si asumimos que el modelo coincide con la dinámica real del sistema, esto es, asumimos que $A_{m}=A_{p}, B_{m}=B_{p}$ and $C_{m}=C_{p}$, entonces $\bar{c}=c(T, \ldots, T)$, donde $T=T_{s} / n$.

\subsection{Implementación en sistemas reales}

La proliferación de placas computadoras como Raspberry Pi o Beaglebone Black [32, 7] unido al creciente uso de las redes de comunicación para conectar elementos en un lazo de control implica que el tiempo de computación disponible puede estar limitado. Por esta razón, es importante limitar el esfuerzo computacional del controlador con el fin de garantizar un comportamiento aceptable. En nuestro caso, el algoritmo de optimización puede relajarse de diferentes maneras.

Una fuente importante de computación es la discretización del sistema en lazo cerrado. Dado que (2) implica resolver una integral matricial y dado que una de las variables de la optimización es el límite superior de la integral, el algoritmo de optimización tiene que ejecutar un costoso cálculo en tiempo real para obtener el modelo discretizado en cada iteración. Sin embargo, sabemos el máximo y el mínimo tiempo que puede aplicarse cada señal, ya que debe ser menor que $T_{s}$. Por tanto, podemos considerar un periodo $h$ suficientemente pequeño en función de $n$ y de $T_{s}$ y llevar acabo un cálculo offline de las matrices discretizadas. Es decir, calculamos offline $e^{A(m h)}$ y $B(m h, A)$ para todo $m \in \mathbb{N}$ tal que $0 \leq m h \leq T_{s}$. Así pues, dada esta sucesión de matrices discretizadas, la función de optimización solo necesita acceder al valor requerido en vez de calcular la correspondiente matriz discretizada.

Las restricciones (19)-(20) pueden ser también una fuente de retardos en la computación. Cuando se tiene en cuenta los tiempos de applicación $T_{i}$ en la optimización, debemos consisderar (19)-(20) como restricciones no lineales del problema de optimización, lo que puede suponer un importante esfuerzo computacional si hay un elevado número de señales de control. Para evitar esto, podemos resolver el algoritmo de optimización en dos pasos. El primero proporciona los tiempos de aplicación que optimizan el radio de convergencia para el controlador auxiliar. Con estos tiempos de aplicación fijados, (19)-(20) se transforman en límites constantes para la segunda parte del algoritmo de optimización, que obtiene los valores de la señal de control. Naturalmente, esto resulta en una solución subóptima del problema. A pesar de ello, la solución siempre mejora el desempeño obtenido con el controlador auxiliar.

\section{RESULTADOS EXPERIMENTALES}

En esta sección presentamos los resultados experimentales obtenidos con el algoritmo de optimización. Para ello, hemos utilizado el sistema de levitación por aire propuesto en [6].

En el sistema las únicas fuerzas que actúan sobre el objeto a levitar son el efecto hacia arriba de la corriente de aire y el efecto hacia abajo de la gravedad. De acuerdo con esto, la segunda ley de 
Newton establece el siguiente modelo no lineal

$$
m \Delta \ddot{z}(t)=\frac{1}{2} C_{d} \rho S\left(v_{w}-\dot{z}(t)\right)^{2}-m g,
$$

donde $m$ es la masa del objeto a levitar, $z$ es la posición vertical del objeto en el tubo, $\rho$ es la densidad del aire, $S$ es el área del objeto expuesta al flujo de aire, $v_{w}$ es la velocidad del aire en el interior del tubo, $g$ es la aceleración gravitacional, y $C_{d}$ es el coeficiente de arrastre.

Siguiendo [6], el modelo linealizado de (21) viene determinado por las matrices

$$
\begin{aligned}
A_{p} & =\left(\begin{array}{ccc}
-8,52 & -2,97 & 0 \\
4,00 & 0 & 0 \\
0 & 1,00 & 0
\end{array}\right), B_{p}=\left(\begin{array}{c}
2,00 \\
0 \\
0
\end{array}\right), \\
C_{p} & =\left(\begin{array}{lll}
0 & 0 & 3,36
\end{array}\right), D_{p}=0 .
\end{aligned}
$$

Para resolver el problema de optimización, hacemos uso de este modelo lineal. Como controlador auxiliar, consideramos un controlador PI con ganancias $K_{p}=0,006$ y $K_{c}=0,002$. Además, consideramos un periodo de muestreo de la salida $T_{s}=300 \mathrm{~ms}$ y $n=2$, i.e., la señal de control se cambia dos veces en cada $T_{s}$. Aplicando el teorema 3.2 , se obtiene $\hat{\alpha}=0,0017$ y $\hat{c}=734,24$.

Con estos datos, llevamos a cabo dos experimentos en el sistema real. En la figura 2, se observan los resultados obtenidos con el algoritmo propuesto frente al controlador PI y frente al algoritmo de optimización considerando constantes los tiempos de aplicación. En el experimento, la señal de referencia se fija en $18 \mathrm{~cm}$ y posteriormente se realizan dos cambios. A los $60 \mathrm{~s}$ se fija la referencia en 28 cm, y a los $100 \mathrm{~s}$ se introduce una perturbación en el servo. Las señales de control se muestran en la figura 3. Naturalmente, la optimización del radio de convergencia implica una entrada más agresiva. A pesar de las limitaciones inherentes del sistema experimental como la sensibilidad del sensor, perturbaciones o errores de modelo, que contribuyen a complicar la optimización, se observa que el algoritmo propuesto proporciona una mejora durante la estabilización. Para cuantificar esta mejora se ha medido el error integral cuadrático (integral square error, ISE) y el tiempo de asentamiento, como se ve en la tabla 1.

En la figura 4, se representa la respuesta del sistema para los tres métodos del experimento anterior y sujeta a diferentes perturbaciones. Específicamente, aplicamos una perturbación en forma de diente de sierra entre 80 y 160 s, y a continuación aplicamos varias perturbaciones de tipo impulso entre 200 y $260 \mathrm{~s}$, como se muestra en la figura 5. Similarmente al primer experimento, el algoritmo de optimización implica un mayor número de cambios en la señal de control (véase la figura 6), que es el precio a pagar por maximizar el radio de convergencia. La tabla 1 recoge las medidas del ISE durante las diferentes perturbaciones.

\section{CONCLUSIONES}

En este trabajo, se propone un algoritmo de optimización para un sistema de control multifrecuencia. El algoritmo tiene en cuenta tanto el valor de la señal de control como su correspondiente tiempo de aplicación, lo que implica una importante mejora del radio de convergencia. Por contra, el tiempo de computación del algoritmo puede ser considerablemente grande dependiendo del número de señales de control y tiempos de aplicación a optimizar. Por ello, se describen algunas técnicas para reducir la carga computacional y poder implementar el algoritmo en placas computadoras. A modo de ejemplo, hemos aplicado el esquema de control a un sistema de levitación por aire.

Futuras líneas de trabajo pueden estar enfocadas en la mejora de los tiempos de computación del algoritmo de manera que sea implementable en sistemas más rápidos. Además, en este trabajo se considera fundamentalmente un esquema bifrecuencia, por lo que su extensión a otras configuraciones multifrecuencia puede resultar interesante.

\section{Agradecimientos}

Este trabajo ha sido financiado por el Ministerio de Economía, Industria y Competitividad (MINECO) bajo los proyectos DPI2012-31303 y DPI2014-55932-C2-2-R.

\section{English summary \\ Optimization in multi-rate control systems}

\begin{abstract}
In this work we consider a multi-rate configuration, where $n$ input signals are computed and applied to the system in each sampling period of the output of the plant. Our objective is maximizing the convergence rate of the system to the desired reference. To this effect, we design an optimization algorithm as function of the $n$ input signals and/or the times of application of these signals. We present experimental results obtained in a air levitation system to verify the validity of the method.
\end{abstract}




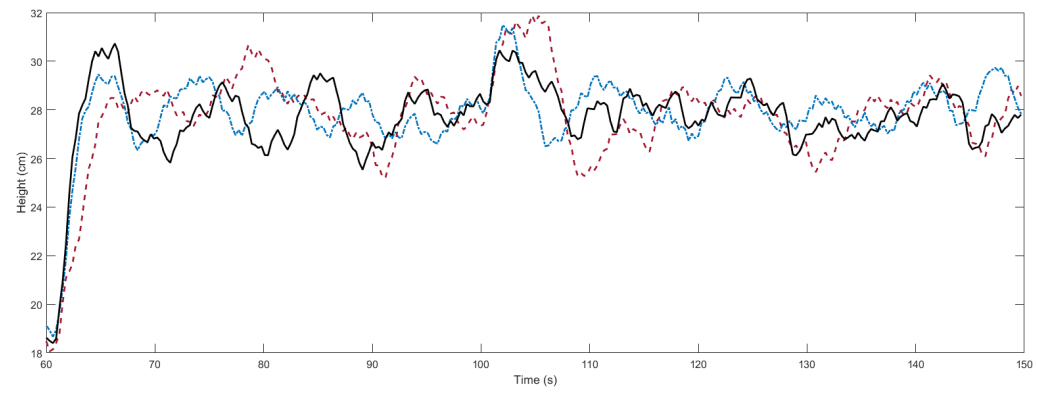

Figura 2: Respuesta de la salida del sistema de levitación por aire en el experimento de estabilización. Línea negra: algoritmo propuesto. Línea azul: algoritmo en [3]. Línea roja: controlador auxiliar PI.
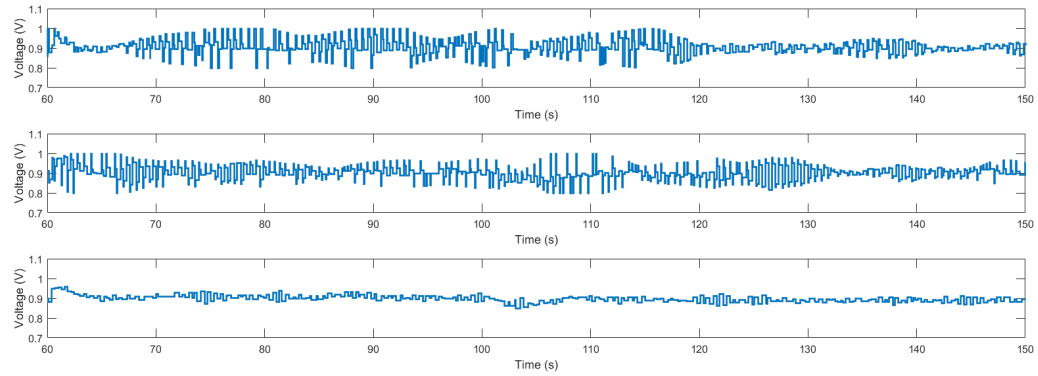

Figura 3: Voltaje de entrada aplicado al sistema de levitación por aire en el experimento de estabilización. De arriba a abajo: algoritmo propuesto, algoritmo en [3].

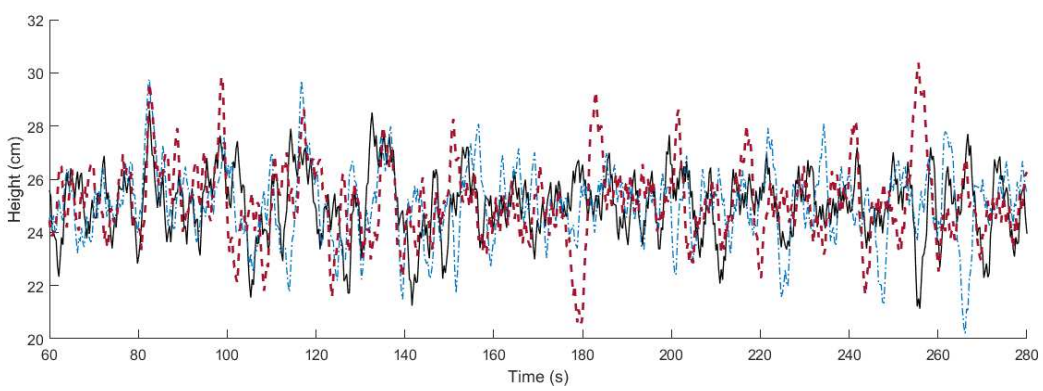

Figura 4: Respuesta de la salida del sistema de levitación por aire en el experimento de rechazo de perturbaciones. Línea negra: algoritmo propuesto. Línea azul: algoritmo en [3]. Línea roja: controlador auxiliar PI.

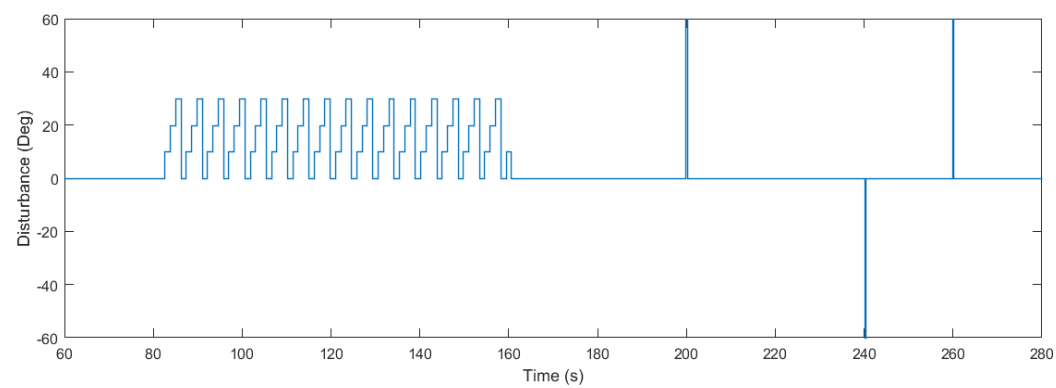

Figura 5: Perturbación aplicada al sistema en el segundo experimento. 


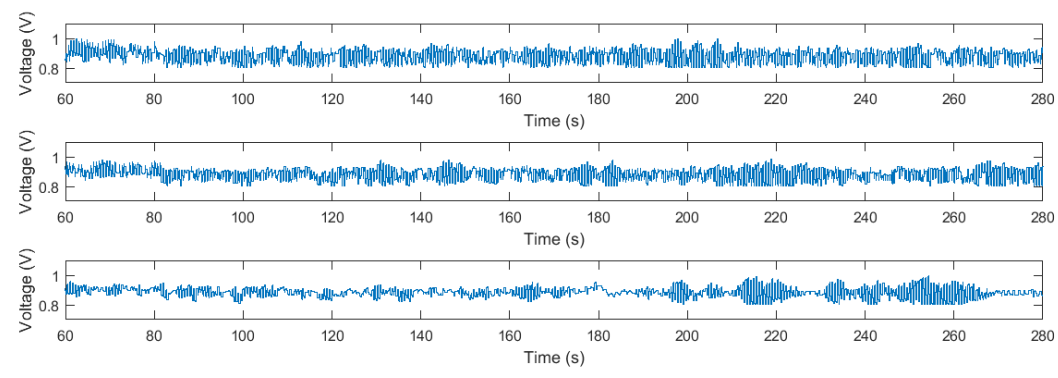

Figura 6: Voltaje de entrada aplicado al sistema de levitación por aire en el experimento de rechazo de perturbaciones. De arriba a abajo: algoritmo propuesto, algoritmo en [3].

Cuadro 1: Comparison of different controllers.

\begin{tabular}{ccccc}
\hline Controlador & $\begin{array}{c}\text { Tiempo de } \\
\text { asentamiento }\end{array}$ & $\begin{array}{c}\text { ISE } \\
\text { (Estabilización) }\end{array}$ & $\begin{array}{c}\text { ISE } \\
\text { (Diente de sierra) }\end{array}$ & $\begin{array}{c}\text { ISE } \\
\text { (Impulso) }\end{array}$ \\
\hline Controlador auxiliar PI & $4,2 \mathrm{~s}$ & 421,39 & 291,01 & 172,43 \\
Algoritmo en [3] & $3,0 \mathrm{~s}$ & 303,78 & 243,64 & 159,03 \\
Algoritmo propuesto & $2,4 \mathrm{~s}$ & 287,53 & 210,54 & 115,51 \\
\hline
\end{tabular}

Keywords: Multi-rate systems, optimization, air levitation system.

\section{Referencias}

[1] Pedro Albertos and Julián Salt. Non-uniform sampled-data control of mimo systems. Annual Reviews in Control, 35(1):65-76, 2011.

[2] Adolfo Anta and Paulo Tabuada. On the minimum attention and anytime attention problems for nonlinear systems. In 49th IEEE Conference on Decision and Control (CDC), pages 3234-3239, 2010.

[3] Ernesto Aranda-Escoástico, Maria Guinaldo, Ángel Cuenca, Julián Salt, and Sebastián Dormido. Anytime optimal control strategy for multi-rate systems. IEEE Access, 5:27902797, 2017.

[4] Bassam Bamieh, J Boyd Pearson, Bruce A Francis, and Allen Tannenbaum. A lifting technique for linear periodic systems with applications to sampled-data control. Systems \&6 Control Letters, 17(2):79-88, 1991.

[5] Sabin-Constantin Carpiuc and Corneliu Lazar. Real-time multi-rate predictive cascade speed control of synchronous machines in automotive electrical traction drives. IEEE Transactions on Industrial Electronics, 63(8):5133-5142, 2016.
[6] Jesus Chacón, Jacobo Sáenz, Luis de la Torre, Jose Manuel Díaz, and Francisco Esquembre. Design of a low-cost air levitation system for teaching control engineering. Sensors, 17(10):2321, 2017.

[7] Gerald Coley. Beaglebone black system reference manual. Texas Instruments, 2013.

[8] Á Cuenca and J Salt. Rst controller design for a non-uniform multi-rate control system. Journal of Process Control, 22(10):18651877, 2012.

[9] Ángel Cuenca, Unnati Ojha, Julián Salt, and Mo-Yuen Chow. A non-uniform multirate control strategy for a markov chaindriven networked control system. Information Sciences, 321:31-47, 2015.

[10] M. C. F. Donkers, Paulo Tabuada, and W. P. M. H. Heemels. Minimum attention control for linear systems. Discrete Event Dynamic Systems, 24(2):199-218, 2014.

[11] YanLi Geng and Bin Liu. Guaranteed cost control for the multi-rate networked control systems with output prediction. In IEEE International Conference on Information and Automation, pages 3020-3025, 2015.

[12] Vijay Gupta. On an anytime algorithm for control. In 48th IEEE Conference on Decision and Control (CDC), pages 6218-6223, 2009.

[13] Xinghui Huang, Ryozo Nagamune, and Roberto Horowitz. A comparison of multirate 
robust track-following control synthesis techniques for dual-stage and multisensing servo systems in hard disk drives. IEEE Transactions on Magnetics, 42(7):1896-1904, 2006.

[14] Rudolf Emil Kalman and J. E. Bertram. General synthesis procedure for computer control of single-loop and multiloop linear systems (an optimal sampling system). Transactions of the American Institute of Electrical Engineers, Part II: Applications and Industry, 77(6):602-609, 1959.

[15] H. K. Khalil. Nonlinear systems (3rd ed.). Prentice Hall, 2002.

[16] Poolla Khargonekar, Kameshwar Poolla, and Allen Tannenbaum. Robust control of linear time-invariant plants using periodic compensation. IEEE Transactions on Automatic Control, 30(11):1088-1096, 1985.

[17] Pramod P Khargonekar and N Sivashankar. H2 optimal control for sampled-data systems. Systems \& Control Letters, 17(6):425-436, 1991.

[18] C. H. Kim, H. J. Park, J. Lee, H. W. Lee, and K. D. Lee. Multi-rate optimal controller design for electromagnetic suspension systems via linear matrix inequality optimization. Journal of Applied Physics, 117(17):17B506, 2015.

[19] J. H. Lee, Marc S. Gelormino, and Manfred Morarih. Model predictive control of multirate sampled-data systems: a state-space approach. International Journal of Control, 55(1):153-191, 1992.

[20] Dongguang Li, Sirish L Shah, Tongwen Chen, and Kent Z Qi. Application of dual-rate modeling to CCR octane quality inferential control. IEEE Transactions on Control Systems Technology, 11(1):43-51, 2003.

[21] Fangzhou Liu, Huijun Gao, Jianbin Qiu, Shen Yin, Jialu Fan, and Tianyou Chai. Networked multirate output feedback control for setpoints compensation and its application to rougher flotation process. IEEE Transactions on Industrial Electronics, 61(1):460468, 2014.

[22] C. Van Loan. The sensitivity of the matrix exponential. SIAM Journal on Numerical Analysis, 14(6):971-981, 1977.

[23] Mahadevamurty Nemani, Tsu-Chin Tsao, and Seth Hutchinson. Multi-rate analysis and design of visual feedback digital servo-control system. Journal of Dynamic Systems, Measurement, and Control, 116(1):45-55, 1994.
[24] Daniel E Quevedo, Wann-Jiun Ma, and Vijay Gupta. Anytime control using input sequences with markovian processor availability. IEEE Transactions on Automatic Control, 60(2):515-521, 2015.

[25] A. J. V. Porto R. K. Mansano, E. P. Godoy. The benefits of soft sensor and multirate control for the implementation of wireless networked control systems. Sensors, 14(12):24441-24461, 2014.

[26] J. Salt, V. Casanova, Á. Cuenca, and R. Pizá. Multirate control with incomplete information over profibus-DP network. International Journal of Systems Science, 45(7):1589-1605, 2014.

[27] Julián Salt and Pedro Albertos. Modelbased multirate controllers design. IEEE Transactions on Control Systems Technology, 13(6):988-997, 2005.

[28] Julián Salt and Masayoshi Tomizuka. Hard disk drive control by model based dual-rate controller. Computation saving by interlacing. Mechatronics, 24(6):691-700, 2014.

[29] Q. M. Shao and A. Cinar. System identification and distributed control for multi-rate sampled systems. Journal of Process Control, 34:1-12, 2015.

[30] T P Sim, G S Hong, and K B Lim. Multirate predictor control scheme for visual servo control. IEE Proceedings-Control Theory and Applications, 149(2):117-124, 2002.

[31] J Tornero, P Albertos, and J Salt. Periodic optimal control of multirate sampled data systems. IFAC Proceedings Volumes (IFAC Workshop on Periodic Control Systems), 34(12):195-200, 2001.

[32] Eben Upton and Gareth Halfacree. Raspberry Pi user guide. John Wiley \& Sons, 2014.

[33] Yunjie Wu, Youmin Liu, and Wulong Zhang. A discrete-time chattering free sliding mode control with multirate sampling method for flight simulator. Mathematical Problems in Engineering, 2013:1-8, 2013.

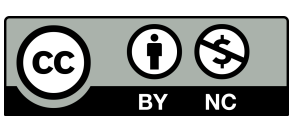
(c) 2018 by the authors. Submitted for possible open access publication under the terms and conditions of the Creative Commons Attribution CC-BY-NC 3.0 license (http://creativecommons.org/licenses/by-nc/3.0/). 